\title{
Fungal $\beta$-Glucan Activates the NLRP3 Inflammasome in Human Bronchial Epithelial Cells Through ROS Production
}

\author{
Yanhua Huang, ${ }^{1}$ Meng Hua, ${ }^{2}$ and Xuefan $\mathrm{Cui}^{2,3}$
}

\begin{abstract}
The nucleotide-binding domain and leucine-rich repeat protein 3 (NLRP3) inflammasome has developed as an important bridge between innate immune and infection recently, and has the ability to drive proteolytic procaspase- 1 into bioactive caspase- 1 , then responsible for proteolytic processing of inflammatory cytokines IL-1 $\beta$ and IL-18. Fungal $\beta$ glucan, a major component of fungal cell wall, triggers inflammatory response in multiple immune cells, but rarely described in epithelial cells. Also, the relationship between fungal $\beta$-glucan and NLRP3 inflammasome is not clear yet. In this study, we first identified that curdlan, a large particulate $\beta$-glucan, could activate the NLRP3 inflammasome in LPS-primed human bronchial epithelial cells (HBECs). RT-PCR and Western Blot showed that curdlan upregulate the mRNA as well as intracellular protein expression of NLRP3 and IL-1 $\beta$ in HBECs, along with the activity of caspase-1, and the level of mature IL-1 $\beta$ in cell supernatants was higher by ELISA detection. Further studies demonstrated that the activation of NLRP3 inflammasome could be attenuated by NAC, an inhibitor of ROS. Thus, it indicated curdlan activate NLRP3 inflammasome through a pathway requiring ROS production in HBECs. These findings may provide a new therapeutic target, NLRP3 inflammasome, in invasive pulmonary fungal infections.
\end{abstract}

KEY WORDS: fungal $\beta$-glucan; NLRP3 inflammasome; ROS; human bronchial epithelial cells.

\section{INTRODUCTION}

Over last two decades, the incidence of invasive fungal infection (IFI) has kept a higher level due to the frequent use of immunosuppressive drugs or the increase of patients with organ transplantation or the growing number of immunocompromised population such as HIV-infected subjects. In clinical, the most common IFIs are invasive candidiasis, invasive aspergillosis, cryptococcosis, and endemic fungi, always cause

\footnotetext{
${ }^{1}$ Qidong People's Hospital, Qidong, China

${ }^{2}$ The First Affiliated Hospital of Nanjing Medical University, Nanjing, China

${ }^{3}$ To whom correspondence should be addressed at The First Affiliated Hospital of Nanjing Medical University, Nanjing, China. E-mail: XuefanC@163.com
}

high intensive care units (ICUs) admissions and mortality rate [1-3]. Unfortunately, there is still no economic and effective treatment so far. Fungal $\beta$-glucans, naturally occurring sugars which possess anti-infective activity, anti-tumor activity, and immunostimulatory activity in vivo or in vitro, consisting of $(1,3)-\beta$-linked glucose polymers with $\beta-1 \rightarrow 6$ linked side chains of varying length and distribution, are important PAMPs mediating the inflammatory reaction and response in the progress of infection [4-6]. Besides, fungal structural framework also includes mannans, proteins, and chitin. Detection of $\beta$-(1,3)-glucan and antibodies to $\beta$-(1,3)-glucan in the plasma of patients are considered as biomarkers of fungal infections, and many other diagnostic tests are also under clinical trials [7,8]. Curdlan, a large particulate $\beta$-glucan, is a pure $\beta$-1,3-glucan that was described to trigger multiple immune cells and experimental animals independent 
of TLRs, instead of relying on dectin-1 receptor, and has been used for many infection-related experiments [9].

Inflammasome is a large protein complex activated upon intracellular or extracellular stimuli. As a large intracellular signaling platform, it generally contains a cytosolic pattern recognition receptor, such as a nucleotide-binding oligomerization domain-like receptor (NLR) or an absence in melanoma2 (AIM2)-like receptor. Among the NLRs, NLRP3 (also known as cryopyrin and NALP3, nucleotidebinding domain, and leucine-rich repeat protein 3 ) is the best characterized one and is found expressed mainly by myeloid lineage cells. It is formed by the assembly of NOD-like receptor NLRP3, ASC (adaptor protein apoptosis-associated speck-like protein containing a caspase recruitment domain), and pro-caspase-1 [10]. And about its activation, although it remains unclear exactly, it is more inclined to be demonstrated as a two-step process, which requires "priming" prior to or coincident with a secondary NLRP3-specific activating signal. The "priming" is usually thought to be the upregulation of NLRP3 via NF-kB pathway, as well as the inflammasomedependent cytokine, pro-IL-1 $\beta$, or pro-IL-18. When it comes to the second step, NLRP3 inflammasome triggers the proteolytic processing of pro-caspase-1 into its active form, caspase-1, which subsequently cleaves pro-IL-1 $\beta$ and proIL-18 into their mature bioactive forms, IL- $1 \beta$ and IL-18,result in various degrees of inflammatory reaction $[12,13]$. The activation of NLRP3 inflammasome is critical in the development of various lung disease, including acute lung injury and acute respiratory distress syndrome, chronic obstructive pulmonary disease, asthma and idiopathic pulmonary fibrosis, sepsis, and respiratory infections. And recently, mounting attention was paid to the role of NLRP3 inflammasome in fungal infection. The airway epithelium, making up the first mechanical barrier of respiratory tract and mediating the initial host immunological response, helps against the invasion of various microbial pathogens. It has been documented that human airway epithelial cells also express NLRP3, though relatively lower than immune cells [14].

In this study, we investigated the effect of fungal glucan (curdlan) on NLRP3 inflammasome in human bronchial epithelial cells and then explore its possible molecular mechanism.

\section{METERIALS AND METHODS}

\section{Reagents}

The immortalized human bronchial epithelial cell line (16HBECs) was obtained from American Type Culture
Collection (ATCC). Optimal concentration for curdlan, Escherichia coli LPS, and NAC (all suspended in PBS) were pretitrated in preliminary experiments, and the final amount used in experiments for curdlan was $100 \mu \mathrm{g} / \mathrm{ml}$ and $1 \mathrm{mg} / \mathrm{ml}$, for LPS was $5 \mu \mathrm{g} / \mathrm{ml}$, and for NAC was 1 and $5 \mathrm{mmol} / \mathrm{L}$. These are all purchased from Sigma-Aldrich. Antibodies against human NLRP3, caspase-1, were from Cell Signaling Technology (MA, USA). Antibodies against GAPDH, IL- $1 \beta$, and $\beta$-tubulin were from Proteintech (Wuhan, China). Human IL-1 $\beta$ Elisa Kit was from R\&D Systems, Minneapolis, MN, USA.

\section{Cells Culture, Infection, and Treatment}

The 16HBEC was cultured with RPMI 1640 (Hyclone) supplemented with $10 \%$ heat-inactived fetal bovine serum (ScienCell), penicillin $(100 \mathrm{U} / \mathrm{ml})$, and streptomycin $(100 \mu \mathrm{g} / \mathrm{ml})$ at $37^{\circ} \mathrm{C}$ under a $5 \% \mathrm{CO}_{2}$ humidified air atmosphere. When reaching 90\% confluence, cells were done the following treatment respectively: (1) (blank control group); (2) LPS (positive control group): LPS ( $5 \mu \mathrm{g} / \mathrm{ml})$ was added directly to cell culture medium for $12 \mathrm{~h}$; (3) LPS + curdlan group: LPS $(5 \mu \mathrm{g} / \mathrm{ml})$ was added to prime cells for $3 \mathrm{~h}$, then remove and add curdlan in different concentrations $(100 \mu \mathrm{g} / \mathrm{ml}$ or $1 \mathrm{mg} / \mathrm{ml})$ for $12 \mathrm{~h}$; and (4) NAC + LPS + curdlan group: NAC in 1 or $5 \mathrm{mmol} / \mathrm{L}$ were added to prestimulate for $1 \mathrm{~h}$ respectively, then LPS $(5 \mu \mathrm{g} / \mathrm{ml})$ primes for $3 \mathrm{~h}$, finally add curdlan $(100 \mu \mathrm{g} / \mathrm{ml}$ or $1 \mathrm{mg} / \mathrm{ml})$ for $12 \mathrm{~h}$.

\section{Total RNA Extraction and Real-Time PCR Analysis}

Total RNA was extracted from 16HBECs using Trizol Reagent (Takara).The concentration and purity of RNA was measured at 260/280 $\mathrm{nm}$ using a NanoDrop spectrophotometer. RNA was temporarily stored at $-80^{\circ} \mathrm{C}$. The $1 \mu \mathrm{g}$ total RNA was reverse transcribed in a $20-\mu 1$ reaction

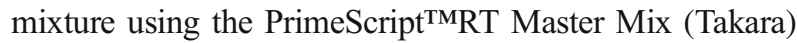
according to the manufacturer's instruction. Real-time PCR was performed in a final volume of $10 \mu$ containing a diluted complementary cDNA solution, $0.2 \mu \mathrm{M}$ of each primer (the primers for NLRP3, IL-1 $\beta$, and GAPDH were as follows: NLRP3 primer: All-in-OneTM qPCR Primers, \#HQP063131, Genecopoeia; CASP1 primer: All-inOneTM qPCR Primers, \#HQP020207, Genecopoeia; IL$1 \beta$ primer: All-in-OneTM qPCR Primers, \#HQP009641, Genecopoeia; GAPDH primer: All-in-OneTM qPCR Primers, \#HQP009640, Genecopoeia); $5 \mu$ of SYBR ${ }^{\circ}$ Premix Ex Taq (Tli RnaseH Plus; 2×); and RNase-free water under the following conditions: 1 cycle at $95^{\circ} \mathrm{C}$ for $30 \mathrm{~s}$, followed by 40 cycles of $95^{\circ} \mathrm{C}$ for $5 \mathrm{~s}$, 
and $60{ }^{\circ} \mathrm{C}$ for $30 \mathrm{~s}$. The relative expression of genes was calculated by the $2^{-\Delta \Delta C T}$ method.

\section{IL-1 $\beta$ Production by Enzyme-linked Immunosorbent Assay (ELISA)}

Cell supernatants were collected at the indicated times and centrifuged at $2000 \mathrm{rmp} 4{ }^{\circ} \mathrm{C}$ for $15 \mathrm{~min}$; aliquots were stored at $-20{ }^{\circ} \mathrm{C}$ until use. The level of IL- $1 \beta$ was assayed by human IL- $1 \beta$ Elisa Kit (R\&D) according to the manufacture's recommendations.

\section{Quantification of Caspase-1 Activity}

The activity of caspase-1 was measured using a Caspase-1 Activity Kit according to the manufacturer's protocol (Beyotime, Shanghai, China). It was based on the principle of active caspase- 1 to cleaving the acetylTyr-Val-Ala-Asp p-nitroanilide (Ac-YVAD-pNA) into p-nitroaniline (pNA), a yellow formazan product. After several steps processing, human bronchial epithelial cell lysates were centrifuged at $12,000 \mathrm{~g}$ for $10 \mathrm{~min}$, and the protein concentrations in supernatant were determined by the Bradford protein assay. Fifty micrograms of protein of each sample were incubated in a 96-well plate with $20 \mathrm{nmol}$ Ac-YVAD-pNA at $37{ }^{\circ} \mathrm{C}$ for $2 \mathrm{~h}$. The absorbance values of pNA at $405 \mathrm{~nm}$ were measured using a 96-well plate reader (BioTek, SantaBarbara, CA, USA). A standard curve of pNA was performed firstly. The pNA in every tested sample was calculated according to the standard curve, and the increase of pNA means the activation of caspase-1.

\section{Measurement of Intracellular ROS}

The generation of intracellular ROS was detected as previously reported. Briefly, after treatment, culture medium was removed. Then, cells were washed with warm PBS three times and detached with Trypsin. Next, cells were resuspended in PBS and incubated with $10 \mu \mathrm{M}$ DCFH-DA (Beyotime, Shanghai, China) at $37{ }^{\circ} \mathrm{C}$ for $30 \mathrm{mins}$, and shake gently every $5 \mathrm{~min}$. Finally, the DCFH-DA fluorescence intensity of 20,000 cells from each sample was measured at an excitation wavelength of $485 \mathrm{~nm}$ and an emission wavelength of $530 \mathrm{~nm}$ using a FACS calibur instrument (Becton Dickinson, San Jose, USA). The relative levels of ROS were quantitatively determined based on the mean fluorescence intensity (MFI).

\section{Western Blot Analysis}

Cells were washed with cold PBS and detached with Trypsin/EDTA. The cell pellets were resuspended in cold lysis buffer supplemented with a complete protease inhibitor PMSF (Beyotime, Shanghai, China) for $20 \mathrm{~min}$ on ice. Lysates were centrifuged at $14000 \mathrm{rmp}$ for $20 \mathrm{~min}$ at $4{ }^{\circ} \mathrm{C}$ to remove debris. Total protein in the supernatant was quantified by the BCA method (Beyotime). Then, $50 \mu \mathrm{g}$ protein of each sample was added and separated by SDSPAGE with electrophoresis, and gel was transferred to PVDF members (Millipore, Billerica, MA, USA).The members were blocked with 5\% non-fat dry milk for $1 \mathrm{~h}$ and then incubated with primary antibodies at $4{ }^{\circ} \mathrm{C}$ overnight, included NLRP3 (1:1000), caspase-1 (1:1000), IL$1 \beta$ (1:500), GAPDH (1:2000), and $\beta$-tubulin (1:1000). After washing three times, the members were incubated with the horseradish peroxidase-conjugated secondary antibodies (BIOSHARP). The bands were developed by the use of ECL reagents (Thermo), and finally detected by a Bio-Rad Gel Doc/Chemi Doc Imaging System. The Quantity One software was used to quantify image densitometry.

\section{Statistical Analysis}

Results were expressed as mean \pm S.D. (standard deviation) from at least three independent experiments. Data were analyzed by one-way analysis of variance for multiple comparisons, and using Student's $t$ test for comparison of two groups. A value of $P<0.05$ was considered as the level of significance.

\section{RESULTS}

\section{Fungal $\beta$-Glucan Upregulates the Expression of NLRP3 Obviously in Human Bronchial Epithelial Cells}

As a kind of fungal glucans, curdlan has a strong proinflammatory effect. However, infection of unprimed human bronchial epithelial cells with curdlan failed to activate the NLRP3 inflammsome; no meaningful changes have occurred on the mRNA of NLRP3 (Fig. 1); this is similar to Hise et al.'s results [15].Then, we choose the curdlan in $100 \mu \mathrm{g} / \mathrm{ml}$ and $1 \mathrm{mg} / \mathrm{ml}$ to combine with LPS priming for next experiments. As Figs. 2 and 6 show, the expression of NLRP3 mRNA and intracellular protein is higher in LPS-primed curdlan group than blank and LPS group, especially in $1 \mathrm{mg} / \mathrm{ml}$ curdlan. 


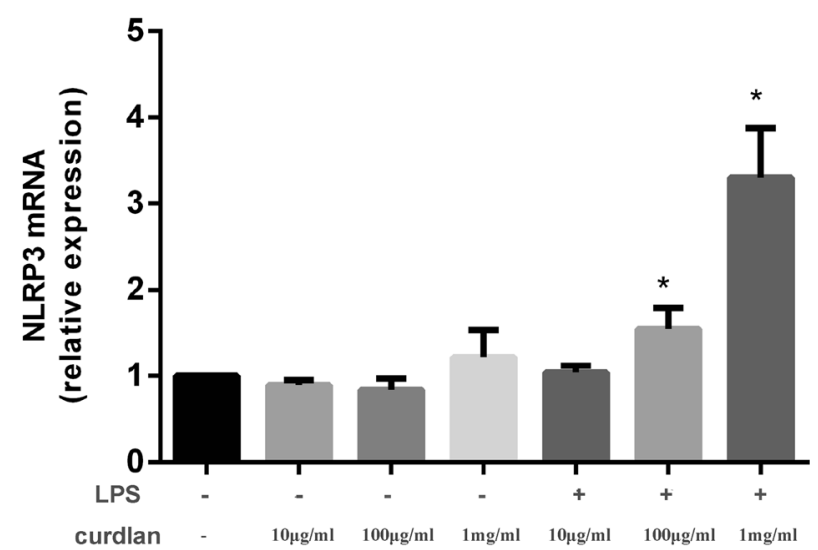

Fig. 1. Effect of curdlan combined with or without LPS on the mRNA expression of NLRP 3 in 16HBECs. In the pre-experiment, the expression of NLRP3 mRNA was not affected by curdlan solely but increased with the increasing of the concentration of curdlan combined with LPSpriming. Values represented as mean $\pm \mathrm{SD}$. ${ }^{*} p<0.05$ compared with the 0 group (blank control group).

\section{Fungal $\beta$-Glucan Increases the Expression of IL-1 $\beta$ Significantly in Human Bronchial Epithelial Cells}

IL-1 $\beta$, as an important inflammatory medium, has been described to participate in various acute and chronic non-infectious or infectious inflammatory diseases. In our study, we used curdlan to co-culture with 16HBECs to mimic pulmonary fungal infection and found that the IL$1 \beta$ mRNA and intracellular protein, as well as the mature IL-1 $\beta$ in cell supernatants were all significantly increased (Figs. 2, 4, and 6); these are all directly proportional to the concentration of the curdlan. It means the fungal glucan evoked the cellular inflammatory responses.

\section{Fungal $\beta$-Glucan Activates Caspase-1 Effectively in Human Bronchial Epithelial Cells}

Caspase-1 is a vital bridge between NLRP 3 and IL-1 $\beta$; therefore, we decided to investigate whether it is activated after LPS priming combined with curdlan stimulation. As demonstrated in Fig. 2, the expression of caspase-1 mRNA was elevated, though slightly. Meanwhile, the results of caspase-1 activity detection showed the cellular caspase-1

Fig. 2. Real-time quantitative RT-PCR analysis on the mRNA expression of NLRP3 (a), caspase-1 (b), and IL-1 $\beta$ (c) after stimulation in 16HBECs. The expression of three different mRNAs all elevated after curdlan combined with LPS priming for indicated time, especially in $1 \mathrm{mg} / \mathrm{ml}$ curdlan, though the increase of caspase-1 mRNA is moderate, and they all decreased gradually when the NAC for pre-stimulation is added. Values represented as mean \pm SD. $* p<0.05$ compared with the 0 group (blank control group), and ${ }^{\#} p<0.05$ compared with the LPS $+1 \mathrm{mg} / \mathrm{ml}$ curdlan group. a

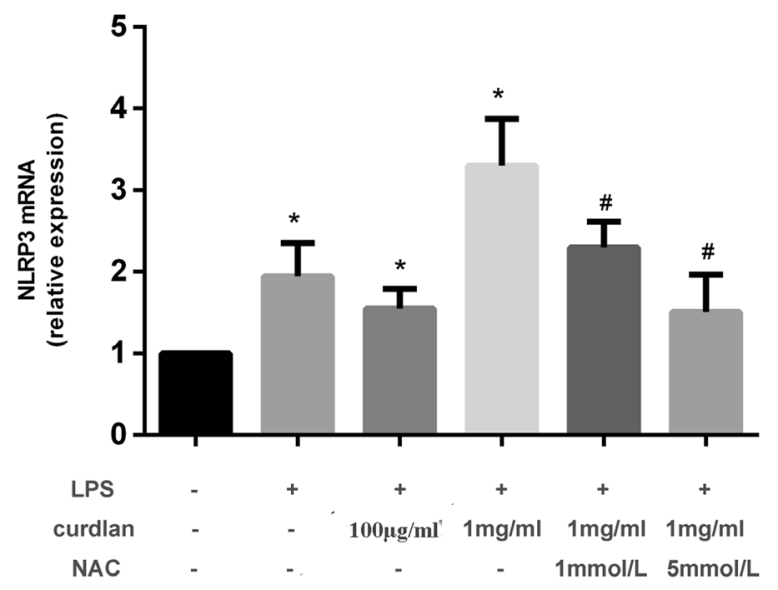

b

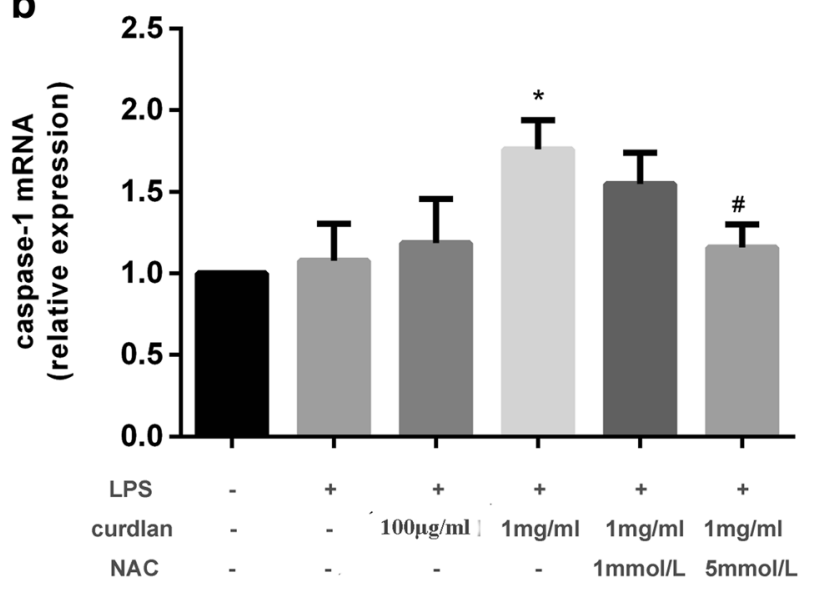

C

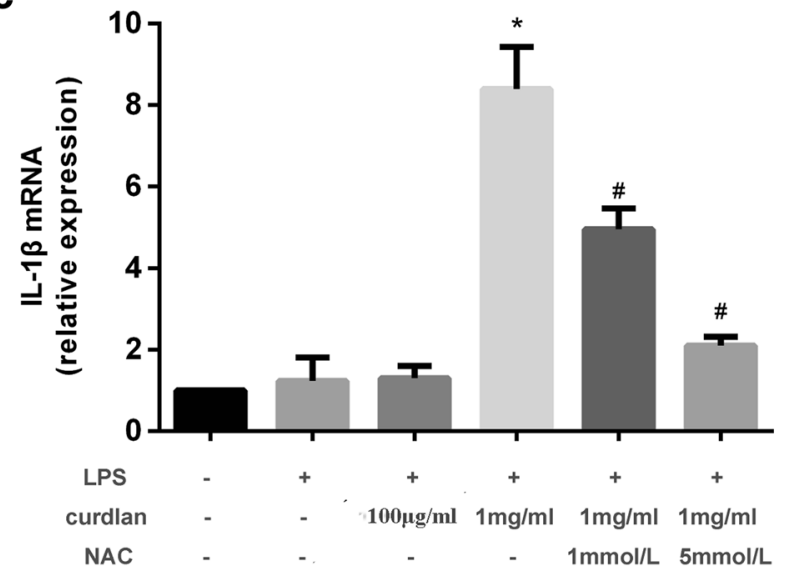

was activated moderately (Figs. 3 and 4); it was consensus with the change of intracellular caspase-1 p20 protein (Fig. 6). These all indicate that NLRP3 inflammasome is triggered by fungal $\beta$-glucan in HBECs. 


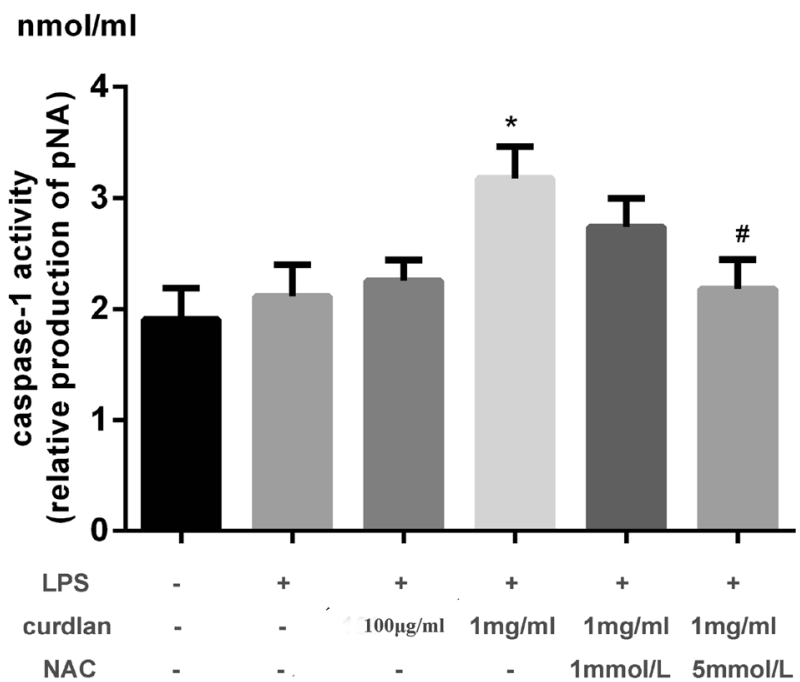

Fig. 3. The caspase-1 activity assay shows the curdlan combined with LPS could activate the caspase-1 moderately in $16 \mathrm{HBECs}$, and this change can be inhibited by NAC.

\section{Reactive Oxygen Species Production Is Essential for Fungal Glucan-Induced Activation of NLRP3 Inflammasome in Human Bronchial Epithelial Cells}

ROS formation has been accepted as a pivotal event in NLRP3 inflammasome activation in many immune cells, such as human macrophages and BMDCs. Hence, we explored whether ROS is required for curdlan-induced

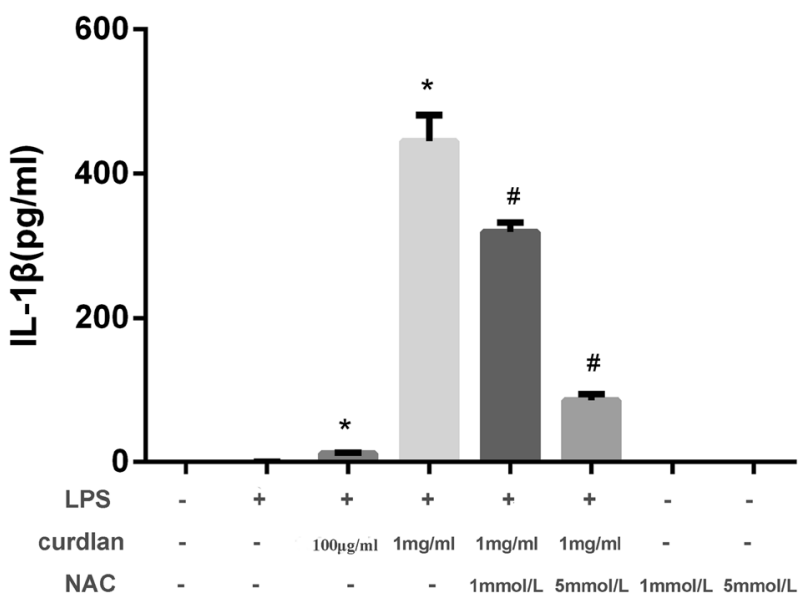

Fig. 4. Competitive ELISA for quantification of IL-1 $\beta$ in cell supernatants after treatments. Curdlan in different concentrations combined with LPS upregulated the IL-1 $\beta$ secretion in 16HBECs and was significant in $1 \mathrm{mg} / \mathrm{ml}$ curdlan. When the cells were pre-incubated with NAC, the level of IL-1 $\beta$ in supernatants was declined sharply, and the NAC alone had no effects on the production of IL- $1 \beta$. Values represented as mean \pm SD. $* p<0.05$ compared with the 0 group (blank control group), and the $\# p<0.05$ compared with LPS $+1 \mathrm{mg} / \mathrm{ml}$ curdlan group.
NLRP3 inflammasome activation in human brochial epithelial cells. We found that curdlan treatment could promote ROS generation markedly as well as in a dosedependent manner. N-acetylcysteine (NAC), an inhibitor of ROS, was added into culture medium for $1 \mathrm{~h}$ prior to stimulation with LPS + curdlan. We observed that NAC, especially in $5 \mathrm{mmol} / \mathrm{L}$, almost abrogated curdlan-induced the raise of intracellular ROS, along with the change of NLRP3, caspase-1, and IL-1 $\beta$ in the level of mRNA, protein, and cytokines, respectively (Figs. 5 and 6).

\section{DISCUSSION}

As an important part of the innate immune system, the NLRP3 inflammasome has emerged as an indispensable immune response platform responsible for the development of various diseases. Up to now, the stimuli known to activate the NLRP3 inflammasome contains numerous pathogens (like bacteria, virus, fungi, and their components), adenosine triphosphate (ATP), pore-forming toxins, and particulate crystals and aggregates. And the possible molecular mechanisms related to the activation of NLRP3 inflammasome signaling pathway have been described briefly, including potassium efflux (a decrease in the intracellular potassium $\left(\mathrm{K}^{+}\right)$concentration), $\mathrm{Ca}^{2+}$ influx (elevated intracellular $\mathrm{Ca}^{2+}$ ), lysosomal destabilization, the generation of cellular ROS (especially those from mitochondria), the release of mitochondrial DNA15 and cardiolipin, spatial arrangement of intracellular organelles, even the upregulation of TXNIP (thioredoxin-interacting protein) and ER stress [10-13, 16].

Given that the prevalence of opportunistic fungal infection has markedly increased worldwide, the researchers are increasingly concerned about the relationship between NLRP3 inflammasome and fungal infections. The role of NLRP3 inflammasome in various fungal infections has been elaborated in many literatures, especially in Candida infection. Several reports have revealed that both early germ tube and fully hyphal forms of Candida are capable of inducing IL- $1 \beta$ release mediating by NLRP3/ASC/Caspase- 1 axis in human PBMC and THP-1 cells, consistent with the results in their mouse mucosal model of Candida challenge [15, 17, 18]. Wellington et al. [19] also showed Candida albicans could trigger pyroptosis in bone marrow-derived macrophages (BMDM) and murine J774 macrophages dependent on caspase-1, ASC, and NLRP3. In Borghi et al.'s study, they not only found that NLRP3 inflammasome activation promoted neutrophil recruitment and inflammation during $C$. albicans infection, but this 

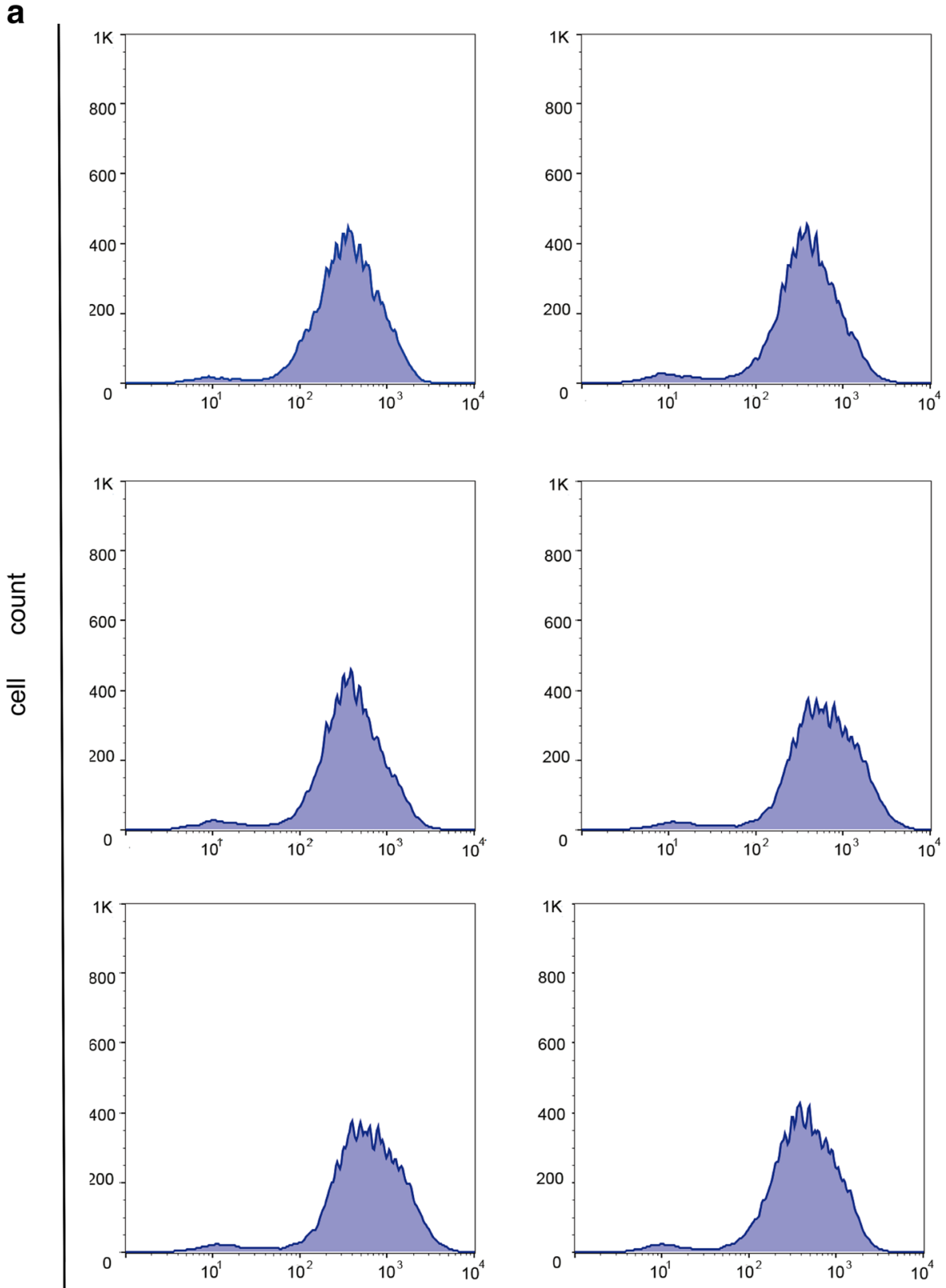

\section{FLH:DCFH-DA}

Fig. 5. Generation of intracellular ROS in human bronchial epithelial cells. Curdlan in $1 \mathrm{mg} / \mathrm{ml}$ combined with LPS could improve the intracellular ROS effectively, but when pre-treat with the ROS inhibitor, NAC, the production of ROS was reduced dramatically. Values represented as mean \pm SD. * $p<0.05$ compared with the 0 group (blank control group), and ${ }^{\#} p<0.05$ compared with the LPS $+1 \mathrm{mg} / \mathrm{ml}$ curdlan group.

activity could be counteracted by IL-22 via activating NLRC4 for sustained production of the IL-1 receptor antagonist IL-1Ra [20]. Moreover, aspergillus fumigatus was demonstrated to induce NLRP3 and AIM2 inflammasome assembly, caspase- 1 and caspase- 8 activation, as well as IL-1 $\beta$ and IL-18 release from immune cells, relying on dectin-1/Syk signaling pathway and $\mathrm{K}^{+}$efflux. ROS production helps host protect against aspergillus infection 


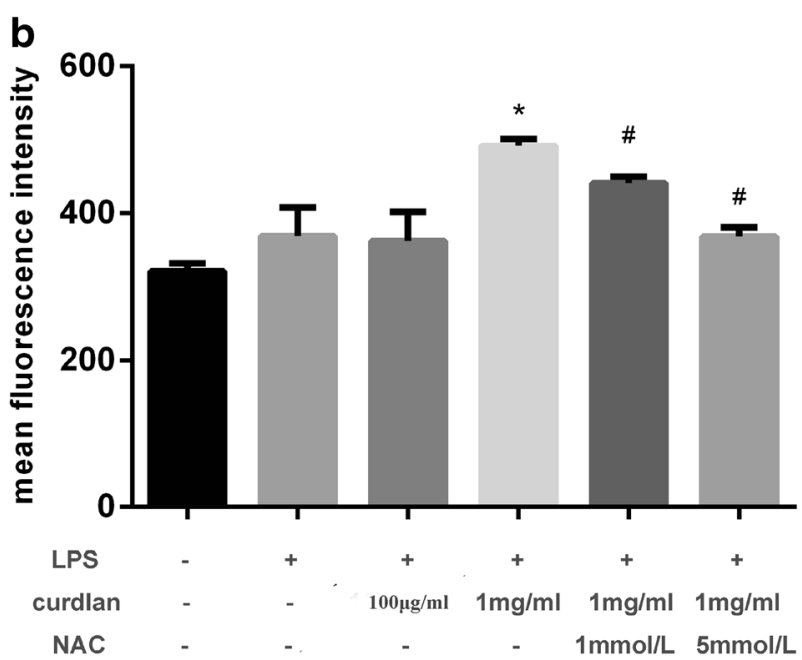

Fig. 5. continued.

$[21,22]$. Additionally, several reports suggested that Cryptococcus neoformans biofilm and the capsular strain instead of C. neoformans yeast lead to ROS production and $\mathrm{K}^{+}$efflux, which result in NLRP3 inflammasome activation, not the AIM2 or NLRC4 inflammasome, and if the encapsular C. neoformans opsonized by anti-GXM$\mathrm{Ab}$ (GXM, glucuronoxylomannan, main component of the capsule), then they were able to activate either the canonica NLRP3-ASC-caspase-1 inflammasome or the noncanonical NLRP3-ASC-caspase-8 inflammasome involving the phagolysosome membrane permeabilization and potassium efflux, and the NLRP3 signaling could help oppose C. neoformans infection [23-25]. Besides, as the major component of fungal cell wall, $\beta$-glucan, has been proved to activate the NLRP3 inflammasome effectively and trigger IL-1 $\beta$ production in immune cells dependent on the dectin-1/Syk signaling pathway, even could activate the dectin-1/ASC/caspase-8/IL-1 $\beta$ pathway, in line with C. albicans infection $[5,9,26]$. In our study, we also found the fungal $\beta$-glucan (curdlan) could trigger IL- $1 \beta$ secretion and NLRP3 inflammasome activation in human bronchial epithelial cells, and like previous researches, reduction of ROS was shown to attenuate the activation of NLRP3 inflammasome.

Furthermore, the NLRP3 inflammasome was also known to be involved in acute lung injury caused by various reasons and lung fibrosis. Jones et al. [27] found the NLRP3, though is not important in neutrophil recruitment or alveolar leakage, it is required for hypoxemia in the development of ALI mediated by LPS plus MV (mechanical ventilation), another report pointed out that NLRP3-deficient mice could suppress inflammatory response to hyperoxia-induced acute lung injury (HALI), and the NLRP3 inflammasome may facilitate a cytokine burst which follows cytokine-dependent inflammation and epithelial cell apoptosis in HALI [28]. Grailer et al. [29] and Han et al. [30] also showed the similar value of NLRP3 in the standard LPS-induced ALI and burn-induced ALI separately. In Lasithiotaki's clinical experiment, the RAUIP patients (rheumatoid arthritis-usual interstitial pneumonia) were detected high levels of IL-1 $\beta$ and IL-18 in their BALF fluid and BALF cells, in line with pre-existing inflammasome activation in these patients, but in IPF patients, the activation of NLRP 3 inflammasome is damaged, as only slightly higher levels of IL-1 $\beta$ in their BALF, guess it is maybe crucial in the high occurrence of IPF's acute exacerbations due to the susceptibility to microbial infections [31]. In other lung inflammatory diseases, patients with the neutrophilic subtype of asthma usually have highlevel expression of NLRP3 inflammasome in their sputum [32]. Kim et al. [33] reported that obesity-associated asthma could be aggravated by inflammation mediated by NLRP3 and IL-1 $\beta$. However, in contrast to previous studies, Besnard et al. [34] proposed the NLRP3 inflammasome may contribute to the control of allergic asthma via enhancing Th17 cell differentiation. Similarly, it still remains controversial between NLRP3 and COPD. Several supported that NLRP3, caspase-1 and IL-1 $\beta$ expression, is increased during the development of cigarette smoke-induced experimental COPD, and NLRP3 inflammasome is essential for the development of COPD [35-38], but then, Di Stefano et al. [39] reported similar expression levels of NLRP3 in the epithelium of COPD and control subjects, they could not detect caspase-1 responses in samples from patients with COPD and nonsmokers or smokers without COPD, even no differences in the expression of IL- $1 \beta$ among them. Briefly, the role of NLRP3 in lung diseases is much complicated, and need better studies and exploration.

Recent data also suggested that the activation of NLRP3 inflammasome related to not only the lung diseases but also the lesions of many other organs. It shows NLRP3 inflammasome can protect mice against infectious inflammatory colitis caused by Citrobacter rodentium, Clostridium difficile, and E. coli, but the caspase-1 activity and levels of IL-1 $\beta$ and IL-18 increased in Crohn's disease were found pro-inflammatory [40]. In liver diseases, the axis of NLRP3-caspase 1-IL-1 $\beta$ promotes the acetaminophen (APAP)-induced liver injury (higher ALT levels, increased neutrophil recruitment, liver failure, and decreased mice survival),as well as ischemia-reperfusion, endotoxin-induced liver injury and cholestasis, alcoholic 


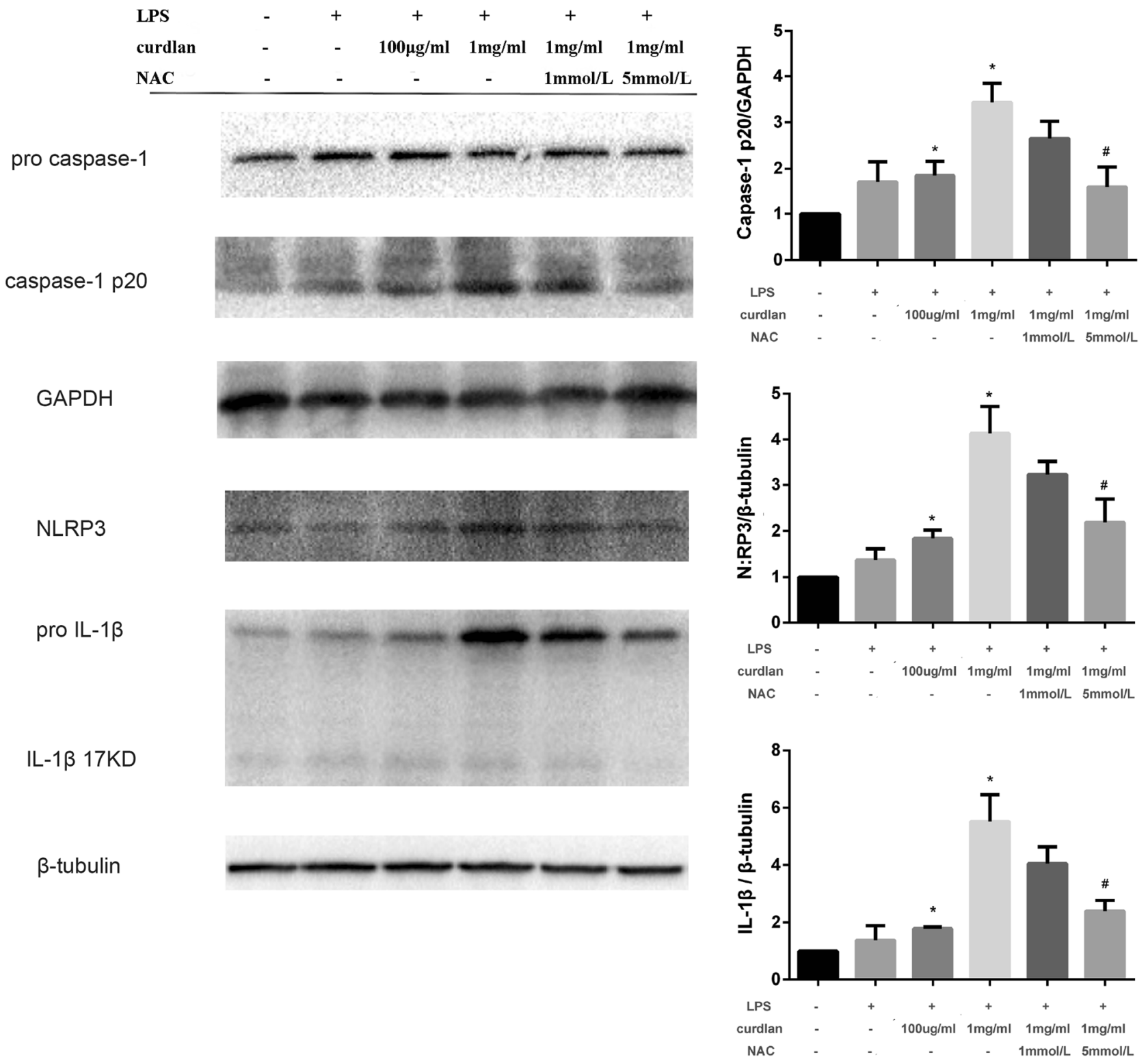

Fig. 6. After treatment, whole-cell lysates were prepared and used for western blot. Curdlan combined with LPS priming increases NLRP3 inflammasomerelated protein, and this process could be suppressed by NAC. Data are shown as mean $\pm \mathrm{SD}$. $* p<0.05$ compared with the 0 group (blank control group), and ${ }^{\#} p<0.05$ compared with the LPS $+1 \mathrm{mg} / \mathrm{ml}$ curdlan group.

and non-alcoholic fatty liver diseases, $\mathrm{HBV}$ and $\mathrm{HCV}$ infection, and liver fibrosis [41]. The elevation in circulating levels of IL- $1 \beta$ also can predict type 2 diabetes when combined with serum IL-6 levels; it demonstrated that increased IL-1 $\beta$ could decrease the insulin sensitivity in mice and contributed to the pathogenesis of chronic kidney disease in both metabolic disorders (least in part, raise the uric acid levels) and autoimmune renal disease (such as
Lupus nephritis) [42], also the atherosclerosis or cardiovascular complications (including cardiac infiltration of phagocytes, inflammatory cytokine levels, infarct size, and myocardial fibrosis and dysfunction) [43, 44].

In summary, this study is the first to demonstrate the relationship between fungal $\beta$-glucan and NLRP3 inflammasome. We found that curdlan could effectively activate the NLRP3 inflammasome and trigger IL-1 $\beta$ 
secretion in human bronchial epithelial cells through intracellular ROS production. Generally, the abnormal activation of inflammasomes can lead to health damage; regulating its activation properly will help control the development of diseases. And our results suggested that the NLRP3 inflammsome may become a potential regulatory target to help fight invasive pulmonary fungal infection in patients. However, our experiments were just limited to cell lines in vitro, not further in vivo(including mice and clinical populations). So, we will try to finish these remaining experiments next, and search drugs or other methods to regulate the activation of NLRP3 inflammasome, to help treat more clinical patients.

\section{ACKNOWLEDGEMENTS}

The authors are grateful to the editor, the associate editor, and the reviewer. This research was supported by Jiangsu Provincial Special Program of Medical Science, China (BL2012012).

\section{REFERENCES}

1. Hayes, G.E., and D.W. Denning. 2013. Frequency, diagnosis and management of fungal respiratory infections. Current Opinion in Pulmonary Medicine 19 (3): 259-265.

2. Martín-Peña, A., M. Aguilar-Guisado, and J.M. Cisneros. 2014. Does the current treatment of invasive fungal infection need to be reviewed? Enfermedades Infecciosas y Microbiología Clínica 32 (8): 523-528.

3. De Pascale, G., and M. Tumbarello. 2015. Fungal infections in the ICU: advances in treatment and diagnosis. Current Opinion in Critical Care 21 (5): 421-429.

4. Shepardson, K.M., and R.A. Cramer. 2013. Fungal cell wall dynamics and infection site microenvironments: signal integration and infection outcome. Current Opinion in Microbiology 16 (4): 385-390.

5. Kankkunen, P., L. Teirilä, J. Rintahaka, et al. 2010. (1,3)- $\beta$-Glucans activate both dectin-1 and NLRP3 inflammasome in human macrophages. The Journal of Immunology 184 (11): 6335-6342.

6. Tsoni, S.V., and G.D. Brown. 2008. $\beta$-Glucans and Dectin-1. Annals of the New York Academy of Sciences 1143 (1): 45-60.

7. Mori, T., H. Ikemoto, M. Matsumura, et al. 1997. Evaluation of plasma $(1 \geq 3)$-D-glucan measurement by the kinetic turbidimetric limulus test, for the clinical diagnosis of mycotic infections. European Journal of Clinical Chemistry and Clinical Biochemistry 35: 553-560.

8. Miyazaki, T., S. Kohno, K. Mitsutake, et al. 1995. Plasma (1 $\geq 3)$ beta-D-glucan and fungal antigenemia in patients with candidemia, aspergillosis, and cryptococcosis. Journal of Clinical Microbiology 33 (12): 3115-3118.

9. Kumar, H., Y. Kumagai, T. Tsuchida, et al. 2009. Involvement of the NLRP3 inflammasome in innate and humoral adaptive immune responses to fungal $\beta$-glucan. The Journal of Immunology 183 (12): 8061-8067.
10. Jo, E.K., J.K. Kim, D.M. Shin, et al. 2016. Molecular mechanisms regulating NLRP3 inflammasome activation. Cellular \& Molecular Immunology 13 (2): 148-159.

11. Elliott, E.I., and F.S. Sutterwala. 2015. Initiation and perpetuation of NLRP3 inflammasome activation and assembly. Immunological Reviews 265 (1): 35-52.

12. Hosseinian, N., Y. Cho, R.F. Lockey, et al. 2015. The role of the NLRP3 inflammasome in pulmonary diseases. Therapeutic Advances in Respiratory Disease 9 (4): 188-197.

13. Bauernfeind, F., A. Ablasser, E. Bartok, et al. 2011. Inflammasomes: current understanding and open questions. Cellular and Molecular Life Sciences 68 (5): 765-783.

14. Hirota, J.A., S.A. Hirota, S.M. Warner, et al. 2012. The airway epithelium nucleotide-binding domain and leucine-rich repeat protein 3 inflammasome is activated by urban particulate matter. Journal of Allergy and Clinical Immunology 129 (4): 1116-1125.

15. Hise, A.G., J. Tomalka, S. Ganesan, et al. 2009. An essential role for the NLRP3 inflammasome in host defense against the human fungal pathogen Candida albicans. Cell Host \& Microbe 5 (5): 487-497.

16. De Nardo, D., C.M. De Nardo, and E. Latz. 2014. New insights into mechanisms controlling the NLRP3 inflammasome and its role in lung disease. The American Journal of Pathology 184 (1): $42-54$.

17. Joly, S., N. Ma, J.J. Sadler, et al. 2009. Cutting edge: Candida albicans hyphae formation triggers activation of the Nlrp3 inflammasome. The Journal of Immunology 183 (6): 3578-3581.

18. Gross, O., H. Poeck, M. Bscheider, et al. 2009. Syk kinase signalling couples to the Nlrp3 inflammasome for anti-fungal host defence. Nature 459 (7245): 433-436.

19. Wellington, M., K. Koselny, F.S. Sutterwala, et al. 2014. Candida albicans triggers NLRP3-mediated pyroptosis in macrophages. Eukaryotic Cell 13 (2): 329-340.

20. Borghi, M., A. De Luca, M. Puccetti, et al. 2015. Pathogenic NLRP3 inflammasome activity during Candida infection is negatively regulated by IL-22 via activation of NLRC4 and IL-1Ra. Cell Host \& Microbe 18 (2): 198-209.

21. Karki, R., S.M. Man, R.K.S. Malireddi, et al. 2015. Concerted activation of the AIM2 and NLRP3 inflammasomes orchestrates host protection against Aspergillus infection. Cell Host \& Microbe 17 (3): 357-368.

22. Saï-Sadier, N., E. Padilla, G. Langsley, et al. 2010. Aspergillus fumigatus stimulates the NLRP3 inflammasome through a pathway requiring ROS production and the Syk tyrosine kinase. PLoS One 5 (4): e10008.

23. Lei, G., M. Chen, H. Li, et al. 2013. Biofilm from a clinical strain of Cryptococcus neoformans activates the NLRP3 inflammasome. Cell Research 23 (7): 965-968.

24. Guo, C., M. Chen, Z. Fa, et al. 2014. Acapsular Cryptococcus neoformans activates the NLRP3 inflammasome. Microbes and Infection 16 (10): 845-854.

25. Chen, M., Y. Xing, A. Lu, et al. 2015. Internalized Cryptococcus neoformans activates the canonical caspase-1 and the noncanonical caspase-8 inflammasomes. The Journal of Immunology 195 (10): 4962-4972.

26. Ganesan, S. 2014. Characterization of anti-fungal inflammasome responses and the role of caspase- 8 in innate immune signaling: a dissertation.

27. Jones, H.D., T.R. Crother, R.A. Gonzalez-Villalobos, et al. 2014. The NLRP3 inflammasome is required for the development of hypoxemia in LPS/mechanical ventilation acute lung injury. American Journal of Respiratory Cell and Molecular Biology 50 (2): 270-280. 
28. Fukumoto, J., I. Fukumoto, P.T. Parthasarathy, et al. 2013. NLRP3 deletion protects from hyperoxia-induced acute lung injury. American Journal of Physiology-Cell Physiology 305 (2): C182-C189.

29. Grailer, J.J., B.A. Canning, M. Kalbitz, et al. 2014. Critical role for the NLRP3 inflammasome during acute lung injury. The Journal of Immunology 192 (12): 5974-5983.

30. Han, S., W. Cai, X. Yang, et al. 2015. ROS-mediated NLRP3 inflammasome activity is essential for burn-induced acute lung injury. Mediators of Inflammation: 2015.

31. Lasithiotaki, I., I. Giannarakis, E. Tsitoura, et al. 2016. NLRP3 inflammasome expression in idiopathic pulmonary fibrosis and rheumatoid lung. European Respiratory Journal 47 (3): 910-918.

32. Simpson, J.L., S. Phipps, K.J. Baines, et al. 2014. Elevated expression of the NLRP3 inflammasome in neutrophilic asthma. European Respiratory Journal 43 (4): 1067-1076.

33. Kim, H.Y., H.J. Lee, Y.J. Chang, et al. 2014. Interleukin-17producing innate lymphoid cells and the NLRP3 inflammasome facilitate obesity-associated airway hyperreactivity. Nature Medicine 20 (1): 54-61.

34. Besnard, A.G., D. Togbe, I. Couillin, et al. 2012. Inflammasome-IL1-Th17 response in allergic lung inflammation. Journal of Molecular Cell Biology 4 (1): 3-10.

35. Li, C., H. Zhihong, L. Wenlong, et al. 2016. The NLRP3 inflammasome regulates bronchial epithelial cell injury and proapoptosis after exposure to biomass fuel smoke. American Journal of Respiratory Cell and Molecular Biology 55 (6): 815-824.

36. Pauwels, N.S., K.R. Bracke, L.L. Dupont, et al. 2011. Role of IL-1 $\alpha$ and the Nlrp3/caspase-1/IL-1 $\beta$ axis in cigarette smoke-induced pulmonary inflammation and COPD. European Respiratory Journal 38 (5): 1019-1028.

37. Yang, W., H. Ni, H. Wang, et al. 2015. NLRP3 inflammasome is essential for the development of chronic obstructive pulmonary disease. International Journal of Clinical and Experimental Pathology 8 (10): 13209.

38. Kinoshita, T., R. Imamura, H. Kushiyama, et al. 2015. NLRP3 mediates NF- $\mathrm{KB}$ activation and cytokine induction in microbially induced and sterile inflammation. PLoS One 10 (3): e0119179.

39. Di Stefano, A., G. Caramori, A. Barczyk, et al. 2014. Innate immunity but not NLRP3 inflammasome activation correlates with severity of stable COPD. Thorax 69 (6): 516-524.

40. Opipari, A., and L. Franchi. 2015. Role of inflammasomes in intestinal inflammation and Crohn's disease. Inflammatory Bowel Diseases 21 (1): 173-181.

41. Szabo, G., and T. Csak. 2012. Inflammasomes in liver diseases. Journal of Hepatology 57 (3): 642-654.

42. Hutton, H.L., J.D. Ooi, S.R. Holdsworth, et al. 2016. The NLRP3 inflammasome in kidney disease and autoimmunity. Nephrology 21 (9): 736-744.

43. Butts, B., R.A. Gary, S.B. Dunbar, et al. 2015. The importance of NLRP3 inflammasome in heart failure. Journal of Cardiac Failure 21 (7): 586-593.

44. Haneklaus, M., and L.A.J. O'Neill. 2015. NLRP3 at the interface of metabolism and inflammation. Immunological Reviews 265 (1): 5362. 